\title{
Caleta El Morro de Talcahuano: Sobrevivencia de una comunidad pesquera al tsunami de 2010 en Chile $^{1}$
}

MATHILDE MOUSSARD HOLZAPFEL

> Universidad de Concepción, Chile. moussard.mathilde@gmail.com

NOELIA CARRASCO HENRÍQUEZ

> Universidad de Concepción, Chile.noeliacarrasco@udec.cl

ENRIQUE ALISTE ALMUNA

> Universidad de Chile. ealiste@uchilefau.cl

FRANCISCO THER RÍOS

> Programa ATLAS Universidad de Los Lagos, Chile. fther@ulagos.cl

CARLOS HIDALGO GARRIDO

> Programa ATLAS Universidad de Los Lagos, Chile. chidalgarrido@gmail.com

Universidad de Valparaíso

Facultad de Arquitectura

Revista Márgenes

Espacio Arte Sociedad

Caleta El Morro de Talcahuano: Sobrevivencia de una comunidad

pesquera al tsunami de 2010 en Chile

Diciembre 2013 Vol. 10 № 13

Páginas 69 a 78

ISSN elec. 0719-4463

ISSN imp. 0718-4034

Recepción: Agosto 2013

Aceptación: Diciembre 2013

\section{RESUMEN}

El presente trabajo grafica a través del conocimiento local la situación vivida en la caleta El Morro de Talcahuano durante el terremoto y tsunami de la madrugada del 27 de febrero de 2010. En este lugar donde la destrucción provocada por el tsunami fue prácticamente total, no se registraron muertes ni desapariciones entre los habitantes; situación más especial aun si consideramos que en sectores cercanos se cuenta una treintena de víctimas fatales y varias desapariciones. A través de una investigación etnográfica se evidencia el rol fundamental jugado por el conocimiento local en el proceso de configuración y activación de mecanismos de sobrevivencia. A partir de prácticas y saberes territoriales en conjunto con aspectos culturales de la comunidad pesquera estudiada, se constituyen respuestas adaptativas capaces de proteger las vidas de una comunidad entera. PALABRAS CLAVE tsunami, mecanismos de sobrevivencia, comunidad de pescadores

\section{Caleta El Morro in Talcahuano: survival of a fishing community at the 2010 tsunami in Chile ABSTRACT}

The present work graphics through the local knowledge of the situation in the Caleta El Morro in Talcahuano for the earthquake and tsunami in the morning of February 27, 2010. In this place where the destruction caused by the tsunami was almost total, deaths or disappearances among the inhabitants; were not recorded more special situation even if we consider that in sectors near is counted thirty fatalities and several disappearances. Through ethnographic research evidenced the fundamental role played by the local knowledge in the process of configuring and activating mechanisms of survival. From practices and local knowledge in conjunction with cultural aspects of the fishing Community studied, are adaptive responses to protect the lives of an entire community.

KEYWORDS

tsunami, mechanisms of survival, fishing community

Caleta El Morro de Talcahuano: Survie d'une communauté de pêcheurs au tsunami de 2010 au Chili

RÉSUMÉ

Le présent travail révèle à travers le savoir local, la situation vécue dans la caleta El Morro à Talcahuano pendant le séisme et le tsunami de la matinée du 27 février 2010. Dans ce lieu où les destructions causées

Caleta El Morro de Talcahuano: Sobrevivencia de una comunidad pesquera al tsunami de 2010 en Chile $>$ Mathilde Moussard Holzapfel, Noelia Carrasco Henríquez, Enrique Aliste Almuna, Francisco Ther Ríos, Carlos Hidalgo Garrido
Revista Márgenes N 13 Vol. 10 > Diciembre $2013>69$ Facultad de Arquitectura > Universidad de Valparaíso 
par le tsunami furent quasi totales, il n'a pas eu de décès ni de disparition; situation très particulière si l'on considère que dans les secteurs voisins furent recensés trente morts et plusieurs disparitions. Par le biais de la recherche ethnographique, nous avons mis en évidence le rôle fondamental joué par les connaissances locales dans le processus de configuration et l'activation des mécanismes de survie. À partir de pratiques et des connaissances territoriales en conjonction avec les aspects culturels de la communauté de pêcheurs étudiée, apparaissent des réponses adaptatives capables de protéger la vie de toute une communauté.

MOTS CLÉS

tsunami, mécanismes de survie, communauté de pêcheurs

\section{INTRODUCCION}

La complejidad del riesgo y sus alcances son temas presentes en la Antropología desde inicios del siglo XX y han sido estudiados crecientemente en nuestro continente. Los riesgos que han desencadenado los desastres, tragedias o catástrofes que a lo largo de la historia hemos considerado naturales, han acompañado a la humanidad en todo momento, pero es a partir de la segunda mitad del siglo XX que el tema se vuelve relevante para las ciencias sociales al incorporar aspectos como vulnerabilidad, grado de exposición, capacidad de reacción, entre otros (Briones, 2005; Evans, 1994; García Acosta, 2004; Konrad, 1985, 1996; Luhmann, 1996; entre otros). La Organización de Naciones Unidas (ONU), declara, para los años comprendidos entre 1990 y 1999, el Decenio Internacional para la Reducción de los Desastres Naturales, evidenciando un gran interés por controlar las magnitudes y daños de los fenómenos de la naturaleza que amenazan a las poblaciones humanas. Pese a que los países de Latinoamérica adhirieron a esta instancia y realizaron esfuerzos por alcanzar el objetivo, ... el avance en la aplicación de medidas preventivas y de mitigación ha encontrado escollos de toda naturaleza... (CEPAL, 2005:27), habiéndose podido avanzar más que nada en la atención a desastres pequeños y de mediana escala. Los esfuerzos, en general, se han dirigido a mejorar los mecanismos de respuesta ante ocurrencias eventuales y desastrosas, restando interés al entendimiento de las causas y orígenes de los riesgos naturales, a atender la vulnerabilidad de forma anticipada y a conocer la cultura ciudadana en torno a estas temáticas. Si entonces nos preguntásemos qué se puede hacer en torno al objetivo de reducir los daños y pérdidas, Farreras, Ortiz y Alcalá (2003) responden claramente: construcción de mapas de riesgos y la identificación de componentes de la comunidad y del medio ambiente que puedan resultar claves para la articulación de mecanismos que permitan enfrentar adecuadamente una amenaza detonada. Subrayamos entonces el potencial rol de las ciencias sociales y la antropología en la tarea de la visibilización de los componentes de la relación socio-ambiental que, desde la cultura local, dan forma a la apropiación y construcción territorial y de un conocimiento local sobre el que se establecen respuestas adaptativas ante amenazas y desastres.

Chile es un país sísmico. Existen testimonios y registros de terremotos y tsunamis desde la época de conquista y aun así demostró en pleno siglo XXI no estar preparado para enfrentar este tipo de eventos. Al menos no a nivel institucional. De acuerdo a Astroza et al. (2010), los eventos ocurridos la madrugada del 27 de febrero de 2010 a lo largo de la zona centro-sur de Chile afectaron a prácticamente el 79,5\% de la población nacional (de acuerdo a datos del CENSO 2002), y el movimiento sísmico principal ocurrido a las 03:34 de la madrugada (UCT-3) habría alcanzado una magnitud de $8.8^{\circ}$ en la escala de Richter, cobrando un total de 525 vidas (Gobierno de Chile, 2011). Resulta curioso constatar que del total de víctimas sólo un pequeño número corresponda a miembros de comunidades pesqueras, las que normalmente viven en contextos de mayor exposición a las diversas amenazas marítimas. Se estima que entre 75.000 y 80.000 fueron las personas que enfrentaron el tsunami en este contexto, de las cuales sólo 8 fallecieron producto de éste último (Marín et al., 2010), 2 de ellas en la región del BíoBío (CONAPACH, 2010). Esta situación de sobrevivencia generalizada en las comunidades de pescadores creemos se debe a que sus miembros pudieron reaccionar a tiempo, activando mecanismos sociales de sobrevivencia. Esto ocurrió también en la caleta El Morro de la ciudad de Talcahuano donde, tras solo 15 minutos de ocurrido el terremoto, prácticamente la totalidad de sus habitantes se encontraba a resguardo en el cerro contiguo al asentamiento.

Dado este escenario, esta investigación se plantea la siguiente interrogante: ¿se articularon mecanismos locales que permitieron la sobrevivencia de las más de 600 personas que componen la comunidad? De ser así, cuáles y de qué manera. Creemos, a modo de hipótesis, que la respuesta reside en el conocimiento local como factor relevante en la evaluación de riesgos y amenazas, e impulsor de mecanismos efectivos de reacción y protección de la población en la caleta El Morro de Talcahuano. La sobrevivencia total de la población de esta antigua Caleta de pescadores dedicados a la pesca artesanal e industrial no es azarosa y puede ser explicada a través de la configuración territorial articulada con prácticas y conocimientos entendidos como mecanismos de sobrevivencia altamente efectivos y que han sido puestos a prueba varias veces en el pasado.

\section{CARACTERIZACIÓN ÁREA DE ESTUDIO}

Caleta El Morro se ubica en la comuna de Talcahuano, Región del Bío-Bío. Pese a no contar con información desagregada para la caleta, sí es posible ofrecer información comunal y regional para facilitar la contextualización demográfica, ecológica y socioeconómica de caleta El Morro.

La región del Bío-Bío se ubica entre los $36^{\circ} 00^{\prime}$ y $38^{\circ} 30^{\prime}$ de latitud sur y entre los $71^{\circ} 00^{\prime}$ de longitud Oeste y el borde costero que limita con el Océano Pacífico. Con una superficie de $37.068,7$ km² y una población de 1.861 .562 habitantes $^{2}$, destaca por una economía primaria enfocada en la industria forestal y pesquera y una fuerte nucleación humana en el Ilamado "Gran Concepción”, un área urbana de conurbación de 10 comunas. Una de éstas es Talcahuano. Con una población proyectada para el 2011 de 171.584 habitantes y una superficie de $92,3 \mathrm{~km}^{2}$, destaca por su importante actividad portuaria, siderúrgica y pesquera (Ver Tabla $N^{\circ} 1$ ). Destaca también por su constitución geomorfológica. Con presencia tanto en la Bahía de San Vicente y en la Bahía de Concepción, encuentra algunos importantes hitos naturales como el humedal Rocuant-Andalién (amenazado, sin embargo, por el crecimiento urbano del Gran Concepción: Smith \& Romero, 2009), los canales
70 > Revista Márgenes № 13 Vol. 10 > Diciembre 2013: 69-78 Facultad de Arquitectura > Universidad de Valparaíso
$>$ ISSN electrónico 0719-4436 > ISSN impreso 0718-4034 
Ifarle y El Morro, la marisma de Lenga, los cerros Los Perales, San Martín y San Miguel, entre otros.

Tabla $\mathrm{N}^{\circ} 1$. Número de trabajadores por rama de actividad en comuna de Talcahuano

\begin{tabular}{|c|c|c|c|}
\hline \multirow{2}{*}{ Origen } & \multicolumn{3}{|c|}{ Comuna } \\
\hline & 2006 & 2008 & 2010 \\
\hline $\begin{array}{l}\text { Agricultura, ganadería, caza y } \\
\text { silvicultura }\end{array}$ & 1.203 & 1.250 & 979 \\
\hline Pesca & 5.412 & 6.386 & 4.937 \\
\hline $\begin{array}{l}\text { Explotaciones de minas y can- } \\
\text { teras }\end{array}$ & 40 & 36 & 42 \\
\hline $\begin{array}{l}\text { Industrias manufactureras no } \\
\text { metálicas }\end{array}$ & 3.802 & 2.639 & 2.499 \\
\hline $\begin{array}{l}\text { Industrias manufactureras } \\
\text { metálicas }\end{array}$ & 7.980 & 8.815 & 7.064 \\
\hline $\begin{array}{l}\text { Suministro de electricidad, gas } \\
\text { y agua }\end{array}$ & 149 & 214 & 197 \\
\hline Construcción & 10.931 & 10.907 & 9.070 \\
\hline $\begin{array}{l}\text { Comercio al por mayor y menor, } \\
\text { repuestos, vehículos, automoto- } \\
\text { res/enseres domésticos }\end{array}$ & 3.699 & 4.209 & 3.476 \\
\hline Hoteles y restaurantes & 821 & 999 & 1.291 \\
\hline $\begin{array}{l}\text { Transporte, almacenamiento y } \\
\text { comunicaciones }\end{array}$ & 6.757 & 7.500 & 6.665 \\
\hline Intermediación financiera & 422 & 331 & 485 \\
\hline $\begin{array}{l}\text { Actividades inmobiliarias, empre- } \\
\text { sariales y de alquiler }\end{array}$ & 6.071 & 6.961 & 6.494 \\
\hline $\begin{array}{l}\text { Administración pública y defensa, } \\
\text { planes de seguridad social afilia- } \\
\text { ción obligatoria }\end{array}$ & 3.387 & 3.227 & 3.648 \\
\hline Enseñanza & 1.190 & 1.021 & 1.001 \\
\hline Servicios sociales y de salud & 2.924 & 3.270 & 3.542 \\
\hline $\begin{array}{l}\text { Otras actividades de servicios co- } \\
\text { munitarios, sociales y personales }\end{array}$ & 2.702 & 2.812 & 2.768 \\
\hline $\begin{array}{l}\text { Consejo de administración de } \\
\text { edificios }\end{array}$ & 0 & 0 & 12 \\
\hline $\begin{array}{l}\text { Organizaciones y órganos extrate- } \\
\text { rritoriales }\end{array}$ & 0 & 1 & 1 \\
\hline Sin información & 0 & 2 & 0 \\
\hline Total & 57.490 & 60.580 & 54.171 \\
\hline
\end{tabular}

Fuente: Biblioteca del Congreso Nacional de Chile. Disponible en: http://reportescomunales.bcn.cl/2012/index.php/ Talcahuano\#Indicadores_econ.C3.B3micos

La caleta El Morro es un emplazamiento de más de cien años, según relatos de algunos habitantes (no existen registros oficiales que indiquen con precisión alguna fecha fundacional). Ubicada en la parte de la costa de la Bahía de Concepción en la comuna de Talcahuano y en pleno centro de la ciudad-puerto del mismo nombre, nace de la necesidad de contar con un lugar donde habitar y poder desarrollar la pesca artesanal al resguardo del viento (existe evidencia arqueológica que destaca la importancia de la pesca en los procesos de intensificación de la ocupación en la Bahía de Concepción desde el holoceno medio-tardío (Torres, Silva \& Lucero, 2007). Debido a las favorables condiciones que el territorio ofrece para esta actividad se ha mantenido hasta hoy como una población de pescadores urbanos en la que se desarrollan tanto la pesca artesanal como la industrial. Los aproximadamente 600 habitantes del Morro son en gran mayoría nacidos y criados allí, hijos y nietos de pescadores o de personas que llegaron hace ya muchos años. La comunidad no ha experimentado grandes migraciones a lo largo de su historia por lo que los vínculos familiares juegan un importante rol.

La comunidad de habitantes de la caleta El Morro está constituida por familias extensas, a veces de 4 generaciones, donde la actividad económica que destaca es la pesca artesanal. Si bien las nuevas generaciones de pescadores han incursionado en el área industrial, la mayoría de los pescadores de más edad (sobre 50 años) son artesanales. Las esposas, por su parte, se dedican mayoritariamente a las labores domésticas y de crianza de los hijos. La mayoría de los habitantes son descendientes de algunas familias consideradas fundadoras: Gutiérrez, Vásquez, Alvear, Macaya, Neira, Plasencia y Torres, principalmente. Son familias numerosas vinculadas entre sí a través de matrimonios pero que también han acogido en su seno a quienes han llegado a vivir a la caleta de forma reciente. En la actualidad varios de sus habitantes ostentan dos de los apellidos antes mencionados, dándose un caso especial y anecdótico como el de dos hermanos Macaya casados con dos hermanas Gutiérrez, cuyos 4 hijos, primos entre sí, comparten exactamente los mismos apellidos. El parentesco no sólo determina la pertenencia a la comunidad, sino que también influye en el lugar de residencia de los hijos luego de casarse. Hace ya algunos años la Caleta dejó de expandirse producto del límite que presentan la pesquera SPK, el cerro El Morro, la avenida Pérez Gacitúa y las modificaciones que se le han hecho al canal. Antes de la saturación, quienes necesitaban construir su hogar ocupaban terrenos vacíos o rellenaban el espacio junto al mar en que querían habitar. Hasta el terremoto del 60, por ejemplo, el sector más cercano a la SPK era una poza donde se dejaban las embarcaciones que luego fue rellenado de forma artesanal.

En la actualidad prácticamente no se desarrolla la pesca artesanal de forma independiente en la Caleta, puesto que la mayoría de los hombres dedicados al mar trabajan como tripulantes en barcos pesqueros de armadores que pueden o no ser habitantes del sector. Los recursos que se trabajan de forma permanente en el Morro son el pejerrey (Odontesthes regia), la lisa (Mugil spp.), corvina (Cilus gilberti) y robalo (Eleginops maclovinus) y entre los meses que duró el trabajo en terreno (abril a julio) se encontraba en apogeo la temporada de sardinas (Sardinops sagax). La actividad pesquera en la Región del Bío-Bío y la comuna de Talcahuano destaca a nivel nacional por su importante desarrollo pesquero artesanal e industrial ${ }^{3}$, siendo caleta El Morro un asentamiento habitado principalmente por pescadores artesanales. En cuanto a su importante rol en la actividad pesquero artesanal de Chile, puede apreciarse en la Tabla $N^{\circ} 2$ presentada más adelante, que el Registro Pesquero Artesanal indica que se trata de la región con mayor número de pescadores artesanales y la segunda en cantidad de organizaciones de pesca artesanal. El producto de la pesca en El Morro es mayoritariamente entregado en Coronel a las grandes pesqueras, vendido a clientes habituales como restaurantes o supermercados mientras otra fracción es llevada a los hogares para consumo fa- 
miliar. Los pescadores empleados en la pesca industrial obtienen salarios estables y significativamente superiores a los empleados en la pesca artesanal, por lo que no requieren de actividades complementarias para la generación de ingresos.

$>$ Tabla N². Registro Pesquero Artesanal año 2012

\begin{tabular}{l|r|r}
\hline Región & Organizaciones & Socios \\
\hline Arica y Parinacota & 7 & 485 \\
\hline Tarapacá & 13 & 500 \\
\hline Antofagasta & 34 & 1.202 \\
\hline Atacama & 42 & 1.626 \\
\hline Coquimbo & 71 & 3.869 \\
\hline Valparaíso & 53 & 2.922 \\
\hline O`Higgins & 20 & 623 \\
\hline Maule & 28 & 980 \\
\hline Bío-Bío & 224 & 12.431 \\
\hline Araucanía & 16 & 537 \\
\hline Los Ríos & 53 & 1.888 \\
\hline Los Lagos & 293 & 10.174 \\
\hline Aysén & 80 & 2.387 \\
\hline Magallanes & 8 & 214 \\
\hline Total & 942 & 39.838 \\
\hline & &
\end{tabular}

Fuente: www.sernapesca.cl

\section{ESTRATEGIA METODOLÓGICA}

La estrategia metodológica empleada en la investigación puede definirse como cualitativa y no experimental. La estrategia fue diseñada y aplicada considerando las ventajas de la etnografía como modalidad investigativa en terreno y de su combinación con revisión de fuentes secundarias, particularmente la revisión de literatura socioantropológica e informes técnicos.

Las técnicas de recolección y análisis de información empleadas fueron:

1. Observación directa: gran parte del trabajo se realizó en terreno en jornadas que incluían el acompañamiento de los actores locales en actividades cotidianas como conversaciones, caminatas, comidas, entre otras. Esta técnica permitió el acercamiento a algunos habitantes de la caleta, al establecimiento de vínculos de confianza con éstos y el primer acercamiento a la dinámica social de la cotidianeidad en la caleta;

2. Diario de Campo: en base a los avances del trabajo en terreno y la observación directa, los acontecimientos de mayor relevancia fueron sistemáticamente siendo incorporados en una "bitácora de trabajo" o "registro de observaciones", en el que se vertieron impresiones de la investigadora, acontecimientos, conversaciones informales, entre otras;

3. Entrevistas semi estructuradas y estructuradas: aplicadas en base a puntos de saturación de información y construidas a partir de una malla temática constituyó el eje del instrumento (que algunos casos sufrió alteraciones debido al alto impacto emocional que provocó el mencionar acontecimientos específicos del 27 de Febrero de 2010);

4. Talleres de discusión: utilizados por la investigadora para corroborar el buen entendimiento de los resultados de las entrevistas.
Esta instancia no sólo fue muy positiva para el proceso de investigación sino también para los participantes, pues supuso una instancia de comunicación y aprendizaje intergeneracional entre los habitantes de la caleta;

5. FODA: pese a haber alcanzado el punto de saturación con las otras técnicas empleadas, se temía que la información recolectada no fuera suficiente. Por esta razón se optó por aplicar esta metodología basada en identificar, a través de los mismos actores, las Fortalezas, Oportunidades, Debilidades y Amenazas asociadas a vivir en El Morro. Para tal efecto se destinó un tiempo extra al Taller de Discusión. El trabajo desarrollado en este aspecto fue realizado a través de 2 papelógrafos (uno para aspectos internos o comunitarios y otro para los externos o medioambientales). Para evitar la ampliación temática hacia aspectos que tuvieran poca relevancia en torno al problema de estudio, esta metodología se aplicó de forma inducida siendo dirigida hacia la mención de los aspectos de la comunidad y su entorno en cuanto a sus mecanismos de sobrevivencia;

6. Relatos de Vida: El recurrir a los relatos biográficos de las personas permite acceder a información muy valiosa en cuanto a cómo los entrevistados han vivido y significado los procesos que les han marcado. Para el caso de este estudio, la vivencia de otras emergencias relacionadas con el mar ha dejado huella y ha determinado procedimientos de reacción ante nuevas amenazas. En base al material recolectado a través de las entrevistas y la participación en el taller de discusión, se pudo desarrollar tres relatos de vida, con la intención de evidenciar, en la historia biográfica de los habitantes del Morro, cómo la constante amenaza por parte del mar ha determinado sus respectivas capacidades de reaccionar ante situaciones como las del 27 de febrero de 2010. Los tres relatos corresponden a personas adultas con más de 35 años de residencia en la caleta, que vivieron su infancia y juventud en ella y que sobrevivieron a la situación que marca el eje de esta investigación;

7. Árboles genealógicos: Para comprender de mejor forma cómo se configura la comunidad y definen los vínculos entre los habitantes, se desarrollaron árboles genealógicos de las familias más numerosas y antiguas del sector. Esto permitió establecer de forma más clara las cercanías y distancias sociales existentes entre los morrinos, y fue aplicada para la exploración del rol de las estructuras familiares en los mecanismos de supervivencia activados en los eventos de Febrero de 2010.

\section{APROPIACIÓN TERRITORIAL Y CONOCIMIENTO LOCAL}

La producción del espacio social se entiende a través del peso explicativo de las lógicas de acción y de las estrategias a las que recurran los actores. (Di Méo y Buléon, 2007). Estos autores caracterizan las prácticas sociales de la siguiente manera:

Entendemos por prácticas sociales todos los desplazamientos y frecuentaciones de lugares, todas las acciones espacializadas que el individuo desarrolla en su medio. Estas van desde sus primeros aprendizajes hasta las organizaciones colectivas que él ayuda a forjar y de las que participa concretamente... (2007:37).

Las características de cada comunidad, las relaciones sociales y significaciones que se dan en ellas, dan paso a modelos locales [que] evidencian un apego especial al territorio concebido como una entidad multidimensional que resulta de muchos tipos de prácticas y relaciones (Escobar, 2000:179-180). Entonces, para
72 > Revista Márgenes Nº 13 Vol. 10 > Diciembre 2013: 69-78 Facultad de Arquitectura > Universidad de Valparaíso
$>$ ISSN electrónico 0719-4436
> ISSN impreso 0718-4034 
hablar de territorios y territorialidad se hace necesario, como ya puede parecer obvio, identificar a quienes habitan el espacio en cuestión. Para este propósito y dadas las características de la población que hace uso residencial de la caleta El Morro, recurriremos a la definición de comunidad que presenta Ander-Egg:

Una comunidad es una agrupación o conjunto de personas que habitan un espacio geográfico delimitado y delimitable, cuyos miembros tienen conciencia de pertenencia o de identificación con algún símbolo local y que interaccionan entre sí más intensamente que en otro contexto, operando en redes de comunicación, interés y apoyo mutuo, con el propósito de alcanzar determinados objetivos, satisfacer necesidades, resolver problemas o desempeñar funciones sociales relevantes a nivel local (1998:33-34).

En cuanto a los actores del territorio, Di Méo y Buléon (2007) diferencian entre "endógenos", "exógenos" y "transicionales". Los primeros corresponderían a los actores originarios e impregnados del lugar en que habitan, aquellos que se identifican plenamente y prioritariamente con él. Los actores exógenos, por otro lado, serían quienes se relacionan de manera indirecta con el territorio, generalmente a partir de valores utilitarios y alejados a las cotidianeidades de los actores endógenos. No se identifican con él ni corporal ni afectivamente. En una posición intermedia se identifica al actor "transicional". Para el caso de esta investigación, se trabaja con actores endógenos, que habitan el sector del Morro y forman parte de él, recurriendo a algunos actores transicionales como los encargados de administrar el Campamento y luego la Aldea, o a externos, como representantes gubernamentales. Son estos actores endógenos los que habitan un espacio y configuran un territorio en base a un acervo de experiencias transmitidas intergeneracionalmente y que ...funcionan como un esquema de referencia en forma de "conocimiento a mano" (Baeza, 2000: 39). De acuerdo a Patrice Melé, los actores habitantes de un territorio adquieren saberes respecto de su medio ambiente, confiriéndoles ciertas expertise y que los constituye en actores legítimos de su territorio a partir de prácticas sociales y territoriales realizadas en él (2009:49-53). Estos elementos teórico-conceptuales resultan importantes puesto que consideramos que las prácticas y saberes cotidianos que facilitan la conquista del objetivo de sobrevivencia provienen de la transmisión de conocimiento acumulado de los actores endógenos. El propósito de esta investigación ha sido explorar y examinar estas prácticas y saberes activados la madrugada de Febrero de 2010 en caleta El Morro.

\section{AMENAZAS, VULNERABILIDADES Y RIESGOS}

Antes de la presentación de los resultados, estimamos necesario clarificar algunas definiciones conceptuales que serán claves para la comprensión de este artículo. Para comenzar, entenderemos por amenaza a una ...fuerza o energía potencialmente destructiva que requiere de un detonador para ser liberada (CEPAL, 2005:9) y que plantea la posibilidad de tener que enfrentar dificultades relacionadas a agentes externos y ante las cuales se deberá reaccionar (Giordano y Boscoboinik, 2002:12); podremos comprender que el terremoto de las 3:34 am actuó como detonador de otro fenómeno de la naturaleza que por sí mismo no debería ser comprendido como un desastre, sino sólo a partir del momento en que se articula con vulnerabilidades de un grupo humano y deriva en la generación de un riesgo socioambiental. El concepto de vulnerabilidad es utilizado aquí para hacer referencia a la predisposición o inca- pacidad de un grupo humano para absorber, resistir, el impacto de fenómenos naturales, como un tsunami, por ejemplo. Oliver-Smith destaca en este sentido la importante ...combinación de factores que determina el grado en el cual la vida de alguien y la forma en que la lleva a cabo son puestos en riesgo por un evento discreto e identificable en la naturaleza o en la sociedad (2007:28). Por su parte, un riesgo corresponde a la posibilidad de que una amenaza o fenómeno natural detonado y una situación de vulnerabilidad confluyan en un mismo contexto. Se da como consecuencia de los peligros inherentes a los ambientes físicos, donde las respuestas a él dependen de la personalidad individual de los afectados (Douglas y Wildavsky, 1983:193). Pero, ¿cómo diferenciar entre riesgo y peligro? Niklas Luhmann establece que si bien ambos son daños hipotéticos, el peligro surge de las características ambientales que rodean a una comunidad, es decir, de agentes externos que escapan a todo control por parte de la sociedad. En cambio, se habla de riesgo cuando "el daño eventual es visto como consecuencia de la decisión” (1996:144).Un terremoto o tsunami constituyen por sí mismos un peligro, pero se transforman en riesgos cuando una población decide conscientemente habitar a orillas del mar conociendo la amenaza a la cual se expone.

Los conceptos de desastre y catástrofe (sinónimos), con que identificamos el escenario resultante de los fenómenos naturales ocurridos la madrugada del 27 de febrero, son definidos por Lamarchand como una eventualidad causante de un daño de amplitud mayor, cuyas consecuencias sociales y simbólicas son de orden histórico (2007:75). Lars Clausen (en Giordano y Boscoboinik. 2002:12) aporta a la definición indicando que producen un repentino, rápido y radical cambio social. En definitiva, los fenómenos naturales juegan un rol decisivo como iniciadores de un desastre pero no constituyen la única causa (García-Acosta, 2002 y 2007). Para nuestro caso, el desastre en caleta El Morro lo configuran la destrucción material (casas, embarcaciones, bienes materiales muebles e inmuebles dañados o destruidos), como también la alteración a la dinámica social, cultural y económica.

Resumiendo, la influencia de los factores vulnerabilidad y las prácticas sociales de apropiación y construcción territorial condicionan el comportamiento de las comunidades a lo largo del proceso que envuelve la ocurrencia de un desastre (Oliver-Smith y Hoffmann, 2007; Blaikie et al., 1994; García-Acosta, 2007). Ofrecemos a continuación un esquema conceptual que sintetiza las relaciones entre algunos de los conceptos referidos.

$>$ Figura 1. Esquema de conformación de una situación de desastre

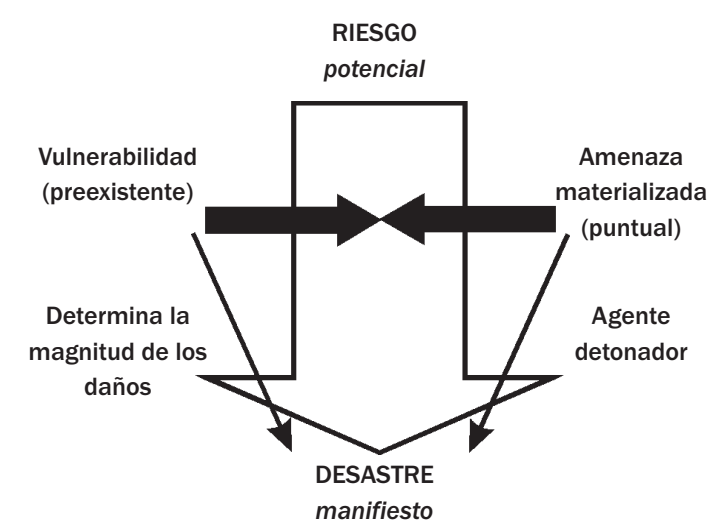

Fuente: Elaboración de la autora 


\section{DE FEBRERO DE 2010: TERREMOTO Y TSUNAM}

Tras el terremoto de la madrugada del 27 de febrero, caleta El Morro fue rápida y espontáneamente evacuada por sus pobladores. Las mujeres abrigaron a los niños y ancianos. Los pescadores, más entendidos en el comportamiento del mar, gritaban por las calles "al cerro, al cerro" indicando al cerro El Morro como vía de escape por si algún vecino no alcanzaba aún a reaccionar. La comunidad completa subió al cerro luego de haber roto la cadena que aseguraba la reja de acceso al Estadio El Morro, recinto deportivo de la Armada que se ubica a un costado de la población y que impide el acceso al cerro. Las familias fueron puestas a resguardo: mujeres, niños, ancianos y hombres no relacionados al mar permanecieron en la altura mientras que los pescadores volvieron a bajar para asegurarse de que no quedaran personas atrapadas y para advertir y avisar sobre el comportamiento del mar. Éste finalmente se recogió, la alerta fue dada, todos los pescadores corrieron y la Caleta quedó vacía: ningún morrino falleció esa noche.

Los hallazgos que a continuación se presentan surgen y se articulan desde un territorio conceptualizado y representado socialmente por sus habitantes, los morrinos, a través de un conocimiento local que ha demostrado (no en una, sino en varias oportunidades) ser de una utilidad y atingencia innegables. A lo largo del tiempo se ha configurado en la Caleta una representación social del territorio común para el grupo que lo habita, elaborada y compartida por la comunidad (Jodelet, 1989:36 en Peretti-Wattel, 2007:200) en base a la situación de riesgo a la que está expuesta.

\section{Arraigo territorial e importancia de las redes sociales}

La comunidad en general manifiesta una importante identidad territorial y apego emocional al modo de vida en la caleta. Se le atribuye una gran importancia al hecho de haber nacido y sido criado allí, y reciben mayor consideración y respeto dentro de la comunidad las personas nacidas y criadas en El Morro. Destaca particularmente las redes de parentesco, ya que la mayoría de los actuales habitantes son descendientes de al menos una de las familias fundadoras. Hablamos de familias extensas y fuertemente ligadas unas con otras, puesto que la historia de la caleta da cuenta de escasos momentos $\mathrm{u}$ oleadas de poblamiento. La solidaridad entre vecinos y el hecho de conocerse desde pequeños facilitan una convivencia amena y desprovista de grandes conflictos entre los habitantes. Destacan, en este sentido, las redes solidarias, el trabajo en equipo de los pescadores y sus familias, y las actividades de recreación y solidarización que hasta antes del último terremoto se realizaban de manera recurrente mediante algunas festividades: Navidad, Año Nuevo, Fiestas Patrias, feriados, celebraciones del sindicato de pescadores o del club deportivo, natalicios y funerales, entre otros.

En general, los morrinos confían en que la comunidad acudirá en su ayuda cuando sea necesario y a la vez están dispuestos a responder de la misma forma cuando corresponda. Esto constituye una de las principales garantías de bienestar brindada por la comunidad a sus miembros:

La gente es unida, y por eso yo no me veo en otra parte que no sea la caleta porque acá te pasa algo y no lo pasas solita con tu familia no más, nopo, aquí lo pasas con toda la gente de la caleta (...) No podríamos estar en otro lugar, por eso estoy acá, porque no me imagino en otro lado, si acá somos todos conocidos, acá los vecinos se cuidan, te cuidan la casa... Cuando hay incendios todos ayudan (Juan Macaya).
Esta solidaridad entre miembros de la comunidad surgió espontáneamente la noche del terremoto cuando varios hombres indicaron a sus familias que corrieran al cerro mientras ellos ayudaban a rescatar a personas atrapadas y también cuando algunas mujeres se hicieron cargo de niños ajenos.

Después que [mi esposo junto con unos vecinos] sacaron a mi mamá él dijo -váyanse para el cerro-Yapo, le dije yo, pero tú tenís que ir. Yél me decía que no podía porque más allá se escuchaba que había gente que gritaba y no podían abrir las puertas. Y yo lloraba y le decía -ipero cómo si tú tenís que irte con nosotros tú siempre hay dicho que somos una familia! (...) Él se fue a ayudar a sacar a una niña que estaba embarazada. La tuvieron que sacar por la ventana, si no pudieron por la puerta, y ella estaba solita, con su pura mamá (Marzury Gutiérrez).

\section{Reconocimiento de la vulnerabilidad y} transmisión de la experiencia

Tanto los habitantes más antiguos como los pescadores han adquirido un conocimiento particular respecto del comportamiento del mar y de las diversas amenazas relacionadas con este elemento del territorio. Ellos, a lo largo de los años, han transmitido la experiencia a las nuevas generaciones con la intención de que éstas conozcan los riesgos asociados al lugar que habitan y puedan reaccionar ante ellos. En la medida en que los morrinos reconocen en el entorno que habitan la exposición a riesgos que son inherentes al territorio, se hacen cargo de la situación. Ellos han desarrollado una respuesta adaptativa acorde con lo que Giddens Ilama el compromiso radical (2002:128-131) y que consiste en el desarrollo de un cuestionamiento y de acciones destinados a la reducción de impactos o de la trascendencia de estos a través de actos racionales.

A lo largo de la historia del asentamiento sus habitantes han adquirido una vasta experiencia respecto del manejo de las diversas emergencias a las que se han visto enfrentados. Hablamos principalmente de amenazas marítimas como inundaciones provocadas por mareas altas con influencia de luna llena o por los fuertes temporales de invierno cuando aumenta también el nivel del canal que pasa por un costado. Otros fenómenos amenazantes considerados en este trabajo son las marejadas y las "subidas de mar" que ocurrieron tras los terremotos de 1960 y 1985, una falsa alerta de tsunami en 2005. Los habitantes de la caleta han racionalizado este tipo de experiencias llegando a la conclusión de que se trata de situaciones inherentes al territorio que habitan. Ante el deseo de continuar viviendo allí, han decidido adaptar su comportamiento y viven en alerta. En una entrevista el pescador Ernesto Neira, consultado respecto de su noción de riesgo en el contexto de la caleta, responde:

Aquí claro que es peligroso vivir, por la cuestión del tsunami, pero un terremoto fuerte es peligroso en cualquier parte (...) Es más, nosotros aquí corremos menos riesgo que muera gente con un tsunami que en otras partes porque nosotros arrancamos todos pal cerro al tiro. ¿Cuánto más se demora la gente? Unos 5 minutos y están todos ahí. Si a nosotros nos enseñaron de chicos: un temblor fuerte y hay que irse para el cerro. En otras partes no tienen el cerro que tenemos nosotros... (Ernesto Neira).

La generaciones mayores han asumido la posibilidad de verse perjudicados por los fenómenos naturales que han reconocido a lo
74 > Revista Márgenes № 13 Vol. 10 > Diciembre 2013: 69-78 Facultad de Arquitectura $>$ Universidad de Valparaíso
> ISSN electrónico 0719-4436 $>$ ISSN impreso 0718-4034 
largo de su vida en el sector y ante esto transmiten su experiencia a las más jóvenes para que nunca sean encontradas. Aquí la conciencia respecto del entorno se vuelve crucial para la sobrevivencia de la comunidad. Abuelos, padres y tíos transmiten desde muy temprana edad sus conocimientos para el desarrollo de capacidades de respuesta. Esto genera un escudo protector (Giddens, 2002:48) que les permite disponerse hacia las diversas amenazas identificadas en el territorio con un menor grado de angustia.

Mi mami decía: no puede salirse la mar mientras que no haya un terremoto o un temblor, ahi hay que arrancar pal cerro. Porque a ella sus abuelitos, sus papás le enseñaron todas esas cosas. Nosotros también lo llevamos en la mente, si hay un temblor fuerte, ffuerte, fuerte!, ahi arrancamos pal cerro. Ahí no más y no bajar más (Francisca Rodríguez).

\section{Resignación y racionalización ante la magnitud de} las fuerzas de la naturaleza

La manera en que un grupo humano entiende los elementos y relaciones de elementos de su medio ambiente, resultan clave al momento de exposición ante amenazas ambientales. Cuando las comunidades enfrentan de forma regular y periódica algún fenómeno natural amenazante desarrollan paulatinamente mejores mecanismos para reaccionar ante ellos. No obstante, existen algunos fenómenos que no se manifiestan con la frecuencia necesaria para que las comunidades que se enfrentan a ellos desarrollen respuestas adaptativas totalmente adecuadas.
En el caso de los morrinos, la exposición sostenida a eventos de la naturaleza como mareas altas e inundaciones por marejadas o tsunamis ha determinado que estos eventos sean comprendidos como algo que escapa a todo tipo de control humano. Tras el desastre, se ha podido apreciar que ellos reconocen una situación de riesgo y que naturalizan las consecuencias de haberlo aceptado. Los miembros de la comunidad, sobre todo aquellos que están directamente relacionados con la pesca, ven en el territorio mucho más que un espacio donde construir sus viviendas: se trata más bien de un espacio significado que ofrece condiciones favorables en términos tanto comunitarios como económicos. Tal como se indicara en entrevistas, se trata de un hábitat, de un territorio que se debe tanto a sus habitantes como estos a su vez de él. En vez de buscar controlar la naturaleza, asumen su realidad de seres humanos insertos en un contexto mayor y desarrollan lo que está a su alcance técnico y sociocultural para protegerse de posibles daños:

Y la gente vuelve a vivir po, si este es su hábitat y se vuelve a acostumbrar a lo mismo. Pase lo que pase se vuelve a acostumbrar igual no más. Es el hábitat y uno tiene que estar ahí (Alfonso Alvear).

La comunidad del Morro identifica en su territorio características favorables y desfavorables, las que aprovecha o de las que se protege, y que mediatizadas por el conocimiento del habitar posibilitan la sobrevivencia en un lugar considerado de riesgo mayor por actores exógenos. La Tabla $N^{\circ} 3$ resume algunos de los aspectos de mayor relevancia que son reconocidos por los morrinos a nivel comunitario y ambiental.

$>$ Tabla $\mathbf{N}^{\circ}$ 3. Aspectos favorables y desfavorables en ámbitos comunitarios y ambientales en torno al habitar en el sector del Morro. Generación propia a partir de la metodología FODA aplicada durante la investigación

\begin{tabular}{|c|c|c|}
\hline Aspectos & Favorables & Desfavorables \\
\hline \multirow{3}{*}{ Comunitarios } & $\begin{array}{l}\text { en la Caleta hay unión, fuerza, empuje, determina- } \\
\text { ción, solidaridad, humanidad, seguridad, bienestar. } \\
\text { Son sentimientos arraigados a la caleta }\end{array}$ & $\begin{array}{l}\text { por el hecho de vivir en una caleta puedes ser } \\
\text { discriminado }\end{array}$ \\
\hline & $\begin{array}{l}\text { es una caleta muy segura, no existe la } \\
\text { delincuencia }\end{array}$ & ¿Algo malo? ¡Los perros! \\
\hline & $\begin{array}{l}\text { es una caleta muy unida } \\
\text { somos todos familia }\end{array}$ & Que son muy buenos para tomar \\
\hline \multirow{3}{*}{ Ambientales } & $\begin{array}{l}\text { es un privilegio para nuestros hijos el tener el mar } \\
\text { cerca para disfrutar de él }\end{array}$ & $\begin{array}{l}\text { vivir aquí en la caleta es correr un riesgo en un } \\
\text { evento de tsunami y terremoto. Según el grado que } \\
\text { sea }\end{array}$ \\
\hline & $\begin{array}{l}\text { estar cerca del cerro cuando pasa un terremoto o } \\
\text { tsunami }\end{array}$ & $\begin{array}{l}\text { tener pesqueras cerca de nuestra caleta porque } \\
\text { contamina el mar }\end{array}$ \\
\hline & $\begin{array}{l}\text { en la caleta hay la oportunidad de aprender a } \\
\text { trabajar en la pesca, conocer el clima, tener una } \\
\text { vista hermosa de la bahía, tener los colegios cerca, } \\
\text { el hospital y el centro }\end{array}$ & $\begin{array}{l}\text { cuando hay temporales podemos correr el riesgo de } \\
\text { subidas de mar } \\
\text { la preocupación de siempre a las altas mareas por } \\
\text { los temporales de los inviernos }\end{array}$ \\
\hline
\end{tabular}

Fuente: elaboración propia.

Caleta EI Morro de Talcahuano: Sobrevivencia de una comunidad pesquera al tsunami de 2010 en Chile > Mathilde Moussard Holzapfel, Noelia Carrasco Henríquez, Enrique Aliste Almuna, Francisco Ther Ríos, Carlos Hidalgo Garrido
Revista Márgenes № 13 Vol. 10 > Diciembre $2013>75$ Facultad de Arquitectura $>$ Universidad de Valparaíso 


\section{DISCUSIÓN: EL CONOCIMIENTO LOCAL COMO HERRAMIENTA DE PREVENCIÓN DE DESASTRES EN LA COMUNIDAD COSTERA DEL MORRO}

El terremoto y tsunami demostraron que la planificación urbana y los dispositivos de emergencias medioambientales de muchas ciudades del borde costero de la zona afectada, incluida Talcahuano, no se encontraban preparados para hacer frente a los eventos de la madrugada del 27 de Febrero de 2010. A lo menos en Talcahuano no se contaba con vías de evacuación expeditas ni protección explícita de la infraestructura ni menos de la población. CEPAL (2005) ha destacado la importancia de identificar las potencialidades, limitaciones y riesgos de los territorios específicos y diseñar sobre esa base la distribución de los asentamientos y las actividades. Recitando el clásico "Risk and Culture" de Douglas y Wildavsky (1983), si no podemos predecir con exactitud la ocurrencia de un desastre, debemos actuar en consciencia de lo que sí sabemos: que ocurrirán y que debemos estar preparados para enfrentarlos si queremos proteger a nuestras comunidades. Por esta razón resulta imperativo el concentrarse en el componente manejable: la vulnerabilidad entendida en un sentido amplio, que incluya mejoras en los instrumentos de ordenamiento del territorio y el reconocimiento del conocimiento local en su diseño. Mejorar la infraestructura, capacitar a la población para autoprotegerse y promover, si es que no se logra evitar la exposición a riesgos, que la convivencia con estos sea un acto consciente y responsable, corresponden a estrategias alternativas de disminución de la vulnerabilidad. Pero sobre todo $y$ tal como diversos organismos internacionales y autores lo han señalado, el control o manejo de los riesgos y desastres debe considerar la memoria compartida de la población en la que se desea implementar este cometido.

En tanto adaptaciones culturales, los mecanismos de respuesta ante amenazas se relacionan con ...[la] innovación y persistencia en la memoria, historia, visión del mundo, simbolismo, flexibilidad de la estructura social, religión y en la naturaleza protectora del folklore y los cuentos populares (Oliver-Smith y Hoffman, 2007:9). Es un proceso que trasciende el presente y que sólo logrará su objetivo a través de una proyección a futuro. Los conocimientos territoriales y los mecanismos de protección de la población que se evidencian en el lugar de estudio constituyen una fuente de futuras estrategias de manejo de amenazas, y su consideración en instrumentos formales de ordenamiento del territorio dependerá en gran medida de su grado de valoración en tanto conocimiento eficaz. Posiblemente en esto reside la capacidad de resiliencia (Folke, 2006) de lo que podríamos considerar el sistema socio-ecológico (SES) del Morro (Berkes, Colding \& Folke, 2001). De ser así se abre una nueva perspectiva y un nuevo motivo para relevar, aún más, el rol de los conocimientos locales, sobre todo si creemos junto a Young et al. (2006), que es en la robustez y en la resiliencia de las que depende el futuro de un SES y su capacidad de respuestas ante perturbaciones. Sin embargo, también resulta altamente pertinente e interesante tomar en cuenta el aporte de Zolli \& Healy (2012), quienes incluso hablan de sistemas robustos pero frágiles (RPF), en alusión a sistemas caracterizados por ser resilientes ante peligros previstos pero también altamente susceptibles ante amenazas no previstas. Zolli y Healy lo ejemplifican mediante el caso del declive de los arrecifes de coral en Jamaica, y pensamos que puede utilizarse en tanto heurística o modelo para interpretar un posible escenario - por cierto no deseado-, en caleta El Morro. Creemos que es el conocimiento local el que juega un papel fun- damental para evitar que nuestro SES termine transformado en un sistema RPF, reconociendo que se origina a partir de las relaciones y adaptaciones co-evolutivas del SES y que deriva en instituciones, prácticas y tecnologías que amplía capacidad de respuesta ante perturbaciones (Kallis \& Norgaard, 2010), en nuestro caso, el riesgo detonado y transformado en amenaza.

\section{CONSIDERACIONES FINALES}

La comunidad del Morro supo cómo reaccionar y proteger las vidas de sus miembros ante la proximidad del tsunami de 2010, pues posee el conocimiento requerido y la capacidad de identificar en cosa de minutos la pronta ocurrencia de este tipo de fenómenos. Este cúmulo de saberes y aptitudes forma parte de la construcción identitaria de quienes forman parte de la comunidad. Identifican elementos amenazantes y protectores y los tienen siempre bajo vigilancia. Ser de la caleta significa conocer el mar, sus cambios y amenazas, saber que el cerro El Morro es el único recurso a mano para ponerse a resguardo ante un tsunami y que todos los vecinos deben ayudarse en el momento de la evacuación.

Los saberes derivados de la práctica de la pesca y la constante observación del mar, ya sea durante las horas de trabajo desde la borda de una embarcación o de forma contemplativa desde la orilla, permiten a los pescadores estimar los frentes climáticos e incluso detectar variaciones mínimas en la marea. Estos hombres, a su vez, enseñan a sus familias en mayor o menor grado a identificar ciertas señales, asegurando que la comunidad posea un conocimiento general respecto del mar y sus distintos comportamientos. Los morrinos poseen fuertes sentimientos asociados al habitar en la caleta y el hecho de que transmitan a las nuevas generaciones qué es lo que deben hacer al identificar tales o cuales señales en él está indicando que existe un reconocimiento de un riesgo y la intención de protegerse de él y sus componentes. La identificación de lugares seguros, vías y formas adecuadas de evacuación, la determinación roles y procedimientos, manifiestan la intención de permanecer en el territorio de forma activa y en un permanente estado de alerta, bajo lo que podemos comprender como un sistema de comunicación con el medio.

El análisis del conocimiento local a través de investigaciones antropológicas ha permitido validar a este mismo como un conocimiento experto adquirido a través de la observación, volviéndolo, por fin, un conocimiento válido, compatible y articulable con los saberes científicos tradicionalmente valorados por nuestra sociedad. El trabajo realizado en torno a la comunidad pesquera del Morro puede ser un aporte en la incorporación de conocimientos locales al diseño e implementación de sistemas de gestión de riesgo para ciudades de borde costero de nuestro país ante amenazas de tsunami. Poblaciones informadas y concientizadas respecto de los riesgos a los que se exponen estarán mejor capacitadas para enfrentarse a los fenómenos naturales que las amenazan permitiendo una mejor respuesta ante estos tanto a nivel local como nacional.

\section{BIBLIOGRAFÍA}

ASTROZA et al (2010) Intensidades sísmicas en el área de daños del terremoto del 27 de febrero de 2010. Departamento de Ciencias Físicas y Matemáticas de la Universidad de Chile, Santiago; en http://www.scribd.com/ doc/36010627/Informe-de-Intensidades-M-Astroza-y-otros
76 > Revista Márgenes Nº 13 Vol. 10 > Diciembre 2013: 69-78 Facultad de Arquitectura > Universidad de Valparaíso
$>$ ISSN electrónico 0719-4436 $>$ ISSN impreso 0718-4034 
BAEZA M. (2000) Los caminos invisibles de la realidad social, RIL Editores, Santiago.

BERKES F., COLDING J., AND FOLKE C. (2001) Linking Social-Ecological Systems. Cambridge: Cambridge University Press.

BOSCOBOINIK A. (2002) "Le Honduras dans l'œil du cyclone" en Constructing Risk, Threat, Catastrophe, Anthropological Perspectives, Giordano \& Boscoboinik ed. Editions Universitaires Fribourg Suisse, Fribourg, páginas 169-178.

BRIONES F. (2005) La complejidad del riesgo: breve análisis transversal. Revista de la Universidad Cristóbal Colón, N²0:9-19.

CEPAL (2005) Elementos conceptuales para la prevención y reducción de daños originados por amenazas socionaturales. Cuadernos de la Cepal N²1, LOM Ediciones, Santiago.

CONAPACH (2010) "Diagnóstico de los Impactos del Terremoto y Tsunami del 27 de febrero de 2010 en la Pesca Artesanal en Chile". Disponible en http://www.conapach.cl/home/ archivos/informe\%20final.doc

DI MÉO G. \& BULEÓN P. (2007) L’espace social, Lecture géographique des sociétés. Armand Colin, Paris.

DOUGLAS M. (1996) La Aceptabilidad del riesgo según las ciencias sociales.

DOUGLAS M. y WILDAVSKY A. (1983) Risk and Culture. An Essay on the Selection of Technological and Environmental Dangers. Berkeley: University of California Press.

ESCOBAR A. (2000) "El lugar de la naturaleza y la naturaleza del lugar: globalización o posdesarrollo" en Antropología del Desarrollo, Viola A., comp. Ediciones Paidós, Barcelona, páginas 169-216.

(2008) Territories of Difference, Duke University Press, Durham.

EVANS V. (1994) "Percepción del riesgo y noción del tiempo". Desastres y Sociedad N³:7-16. Disponible en: http:// www.desenredando.org/public/revistas/dys/rdys03/dys31.0-oct-24-2001-PRNT.pdf

FARRERAS S., ORTIZ M. y ALCALÁ G. (2003) Riesgo de maremotos en ciudad Lázaro Cárdenas de Michoacán, medidas de prevención. Centro de Investigación y de Educación Superior de Ensenada, México.

FOLKE C. (2006) Resilience: The emergence of a perspective or social-ecological systems analyses. Global Environmental Change, 16:253-267

GARCÍA-ACOSTA V. (2007) "Historical Disaster Research", en Catastrophe \& Culture, The Anthropology of Disaster, Hoffman \& Oliver-Smith ed. School of American Research Advanced Seminar Series, Santa Fe, Nuevo México/Oxford páginas 49-66.

(2004) “La perspectiva histórica en la antropología del riesgo y del desastre. Acercamientos metodológicos" en Relaciones, invierno, año/vol. XXV, número 097, páginas 124-142.

(2002) "Conceptualization and Experiences in Mexican Disaster Research" en Constructing Risk, Threat, Catastrophe, Anthropological Perspectives, Giordano \& Boscoboinik ed. Editions Universitaires Fribourg Suisse, Fribourg, páginas 161-168.

Caleta EI Morro de Talcahuano: Sobrevivencia de una comunidad pesquera al tsunami de 2010 en Chile $>$ Mathilde Moussard Holzapfel, Noelia Carrasco Henríquez, Enrique Aliste Almuna, Francisco Ther Ríos, Carlos Hidalgo Garrido
GIDDENS A. (2002) “Modernidad y autoidentidad" en Las Consecuencias Perversas de la Modernidad, Anthropos Barcelona.

GIORDANO C. y BOSCOBOINIK A. (2002) Constructing Risk, Threat, Catastrophe, Anthropological Perspectives, Giordano \& Boscoboinik ed. Editions Universitaires Fribourg Suisse, Fribourg, 2002 páginas 11-16.

GOBIERNO DE CHILE MINISTERIO DEL INTERIOR (2011) “Informe Final de Fallecidos y Desaparecidos por Comunas", publicado el 31/01/2011 en http://www.interior.gov.cl/filesapp /listado_fallecidos_desaparecidos_27Feb.pdf

HOFFMAN S. (2007) "The Monster and the Mother, The symbolism of Disaster" en Catastrophe \& Culture, The Anthropology of Disaster, Hoffman \& Oliver-Smith ed. School of American Research Advanced Seminar Series, Santa Fe, Nuevo México/Oxford páginas 113-142.

KALLIS G., NORGAARD R. (2010) Coevolutionary ecological economics. Ecological economic, 69: 690-699.

LAMARCHAND F. (2007) Catastrophe, en Diccionaire des Risques, Yves Dupont ed. Armand Colin, Paris 2007, páginas 75-80.

LEY N ${ }^{\circ}$ 18.892. Ley General de Pesca y Acuicultura. Diario Oficial de la República de Chile, Valparaíso, Chile, 28 de Septiembre de 1991.

LUHMANN N. (1996) El Concepto de Riesgo. En: Beriain (comp) Las consecuencias perversas de la modernidad, Anthropos, Barcelona.

MARIN et al. (2010) "The 2010 Tsunami in Chile: Devastation and survival of coastal small-scale fishing communities". Marine Policy (2010), doi: 10.1016/j.marpol.2010.06.010.

MELÉ P. (2009) “Identifier un régime de territorialité réflexive”, en Territories, Territorialité, Territorialization, Controverses et Perspectives, Martin Vanier, ed. Presses Universitaires de Rennes, Francia: 45-55.

OLIVER-SMITH A. y HOFFMAN S. (2007) Catastrophe and Culture, The Anthropology of Disaster, Hoffman \& Oliver-Smith ed. School of American Research Advanced Seminar Series, Santa Fe, Nuevo México/Oxford (2007).

OLIVER-SMITH A. (2007) “Theorizing Disasters, Nature, Power and Culture" en Catastrophe \& Culture, The Anthropology of Disaster, Hoffman \& Oliver-Smith ed. School of American Research Advanced Seminar Series, Santa Fe, Nuevo México/Oxford páginas 23-47.

PERETTI-WATTEL P. (2007) Sociologie du Risque. Armand Colin Editeur, Paris.

SMITH P. \& ROMERO H. (2009) Efectos del crecimiento urbano del Área Metropolitana de Concepción sobre los humedales de Rocuant-Andalién, Los Bastros y Lenga. Revista de Geografía Norte Grande, N 43:81-93

TORRES J., SILVA C., LUCERO M. (2007) El rol de la pesca en la intensificación de las ocupaciones costeras durante el holoceno medio-tardío (bahía de Concepción, Región del Bío-Bío, Chile). Magallania, V. 35 (1):71-93.
Revista Márgenes № 13 Vol. $10>$ Diciembre $2013>77$ Facultad de Arquitectura > Universidad de Valparaíso 
1 Proyecto FONDECYT 1090248 Huellas y dinámicas territoriales: imaginarios del desarrollo y prácticas de transformación del ambiente en el Gran Concepción.

2 Debido a las controversias desatadas tras la publicación del Censo 2012 y sus posibles errores metodológicos, se ha preferido consultar al Censo 2002.

3 La legislación nacional dictada en 1991, Ilamada Ley General de Pesca y Acuicultura, establece una distinción entre pesca artesanal y pesca industrial: la pesca artesanal se define como la actividad desarrollada por personas naturales -o jurídicas, en el caso de las Áreas de Manejo y Explotación de Recursos Bentónicos-, y que se desempeña en una embarcación artesanal, para la que se establece como característica principal el no sobrepasar los 18 metros de eslora y las 50 toneladas de registro grueso; la pesca industrial corresponde a las actividades realizadas por personas en embarcaciones industriales consignadas en la Ley $N^{\circ} 18.892$, Art. 2, Letra N. 